\title{
Optimal Inventory Policy for Deteriorating Items with Seasonal Demand under the Effect of Price Discounting on Lost Sales
}

\author{
Rahul Kumar Pandey, Bindu Vaish \\ ${ }^{1,2}$ Department of Mathematics D. N. College Meerut, India
}

\begin{abstract}
In this paper an inventory model is developed with seasonal quadratic demand and time proportional deterioration. Shortages are considered and are partially backlogged. At the start of shortage period price discount is declared on unit selling price for backordered quantity so as to enhance the demand and to reduce the lost sales which in turn maximizes the total profit per unit time. Numerical illustration and sensitivity analysis of the optimal solution with respect to various parameters is carried out to describe the model.
\end{abstract}

Kew Words: Deterioration, Partial backlogging, Seasonal demand, Price discounting.

\section{Introduction}

Inventory models with constant demand rate have been developed by many researchers in the past. But the demand rate of many commodities may be in dynamic state. This work started with Silver and Meal (1969), who developed EOQ model with time-varying demand rate. Since then many researchers developed inventory models with time varying demand. Khanna and Chaudhari (2003),Manna and Chaudhari (2006), Panda et al (2008) developed an inventory models for perishable products with time varying demand. Skouri et al (2009) presented inventory model with ramp type demand rate, partial backlogging \&Weibull deterioration rate.

Vaish and Garg (2011) formulated an inventory model for non-instantaneous deteriorating items with stock dependent and time decreasing demand. Garg, Vaish and Gupta (2012) developed an inventory model with variable production and linearly increasing demand. Vaish and Agarwal (2012) developed a production inventory model with weibull distribution deterioration, quadratic demand and price discounting during deterioration. Karmarkar and Chaudhary (2014) developed inventory model with ramp type demand and partial backlogging. In any inventory control problem a major factor is the maintenance of inventory of deteriorating items. Many product decay or deteriorate over time. Product such as fruits, vegetables, food stuffs are subject to direct spoilage while kept in store. Further it is common experience that some products deteriorate as soon as they are received in stock. Most of researchers have taken interest in developing inventory models with constant and time proportional deterioration. Ghare and Schrader (1963) were the first to develop an inventory model with an exponential deterioration rate. Then Covert and Philip(1973) generalized Ghare and Schrader's constant exponential deterioration rate to a two-parameter Weibull distribution. Dave and Patel (1981), Philip(1974), Mishra(1975b), Chakarbarty et al (1998), Mukhopadhyayet al (2005), Shah and Acharya (2008), Bhuniaet al (2009), Skouri et al (2009) and many others developed inventory models for time dependent deteriorating items. Mishra (2013) developed an inventory model with instantaneous deterioration, linear time dependent demand and partial backlogging. It has been seen many times that stock ends before the arrival of next replenishment and some customers do not want wait up to the next replenishment. Cheng and Dye (1999) formulated an inventory model with time-varying demand and partial backlogging. Dye et al. (2007) developed an inventory model to find optimal selling price and lot size with variable demand and deterioration rate and exponential partial backlogging. They assumed that backlogging increases exponentially as the waiting time for the next replenishment decreases. Mishra, Singh and Kumar (2013) developed an inventory model with instantaneously deteriorated items with linearly increasing demand and partial backlogging. Karmakar and Chaudhary (2014) developed an inventory model with time proportional deterioration, ramp type demand and partial backlogging. Price discount on unit selling price of goods attracts the customers to buy more and more. The main reasons for discounts include: Firstly, suppliers offer discounts to clear their old stocks and free-up space for the new stock. Secondly, a supplier wants to sell more to make more profits, thirdly, customers are attracted by discounts and they buy more than usual. Further discount offers also reduces the loss due to deterioration for suppliers. Thus price discount is one of the key factors which enhance the demand which in turn increases the total profit per unit time. Ardalan (1994) developed an inventory policy where temporary price discount resulted in increase in demand. Papachristos and Skouri (2003) presented an inventory model for deteriorating item where demand rate is a decreasing function of the selling price. Sana and Chaudhari (2008) presented an EOQ model with price discount offers. Hsu and Yu (2009) proposed an EOQ model under a onetime discount. Panda et al. (2009) developed an EOQ model for deteriorating products with discounted selling price and stock dependent demand. Cardanas-Barron et al (2010) presented an inventory model for determining the optimal ordering policies with advantage of a one-time discount offer and back orders. Vaish, Garg and 
Gupta (2012) presented an inventory model for perishable products with ramp type demand and price discounting. Vaish, B and Agarwal, D (2012) formulated a production lot size model for non-instantaneous deterioration with price discounting and quadratic demand.

The present paper is based on of Mishra, Singh and Kumar (2013) and Garima and Vaish (2011) papers. In Garima and Vaish paper price discount is given on unit selling price before deterioration and after deterioration. The modal is developed for no shortage case while Mishra's paper is developed for linear trend in demand. Shortages were allowed and a constant fraction of the demand was backlogged. Price discount is not given in the model. In the present paper an economic ordered quantity model is developed considering seasonal quadratic type of demand which starts with zero reaches its maximum and ends with zero. Deterioration is instantaneous and time dependent. Shortages are allowed. A fraction of demand is backordered which depends on waiting time up to the next replenishment and a price discount is given on the backordered quantity. Price discount on the backordered quantity reduces the lost sales. The modal is developed for the fixed length of the inventory cycle. Special case of no discount is discussed. Profit maximization technique is used to solve the modal. Numerical illustration, tables and graphs are presented to describe the model. In addition sensitivity analysis of the optimal solution with respect to various parameters involved in inventory problem is carried out.

\section{Assumptions}

1. Demand is quadratic in nature which starts from zero and ends with zero and it follows the pattern $D(t)=$ at $(\mathrm{T}-\mathrm{t})$.Where $\mathrm{a}$ is demand rate and $\mathrm{a}>0$.

2. Shortages are allowed and are partial backlogged. The backlogged rate is described as decreasing function of the waiting time $\frac{1}{1+\delta(T-t)}$ where $(\delta>0)$. Thus a fraction of the demand is backlogged

3. $\mathrm{d}(0 \leq \mathrm{d} \leq 1)$ is the percentage offer on unit selling price on backordered quantity declared at the start of the stock out period. $\alpha=(1-d)^{-n}(n \in R$, the set of real numbers and $n \geq 1)$ is the positive effect of discounted selling price on demand during stock out period. When $d \rightarrow 0, \alpha \rightarrow 1$ i.e. the demand during stocked period will not be increased.

5. The deterioration is instantaneous and is linearly time dependent which is $\theta(\mathrm{t})=\theta \mathrm{t}$.There is no repair or replacement of deteriorating items during the period under consideration.

6. Delivery lead time is zero and cycle length of the inventory model is finite.

\section{Notations}

C Purchasing cost per unit

c Cost of each deteriorated unit

$\mathrm{p} \quad$ Selling price per unit

$\theta \quad$ Deterioration coefficient, $\theta<<<1$

$\mathrm{T} \quad$ Cycle length

$\mathrm{t}_{1} \quad$ The time at which inventory level becomes zero

$\mathrm{Q}_{1} \quad$ Initial inventory level at the beginning of each cycle and

$\mathrm{Q}_{2} \quad$ Backordered quantity

Q Ordered Quantity $\left(\mathrm{Q}_{1}+\mathrm{Q}_{2}\right)$

DQ Deteriorated quantity

h Holding cost per unit

s Shortage cost per unit

$1 \quad$ Lost sale cost per unit

A Ordering cost per order

$\delta \quad$ Rate of backlogging

$\mathrm{R} \quad$ Sales revenue per replenishment cycle

$\mathrm{I}(\mathrm{t}) \quad$ The inventory level at time $\mathrm{t}$.

$F\left(t_{1}, d\right)$ Profit per unit time

$t_{1}{ }^{*}, d^{*}, F^{*}\left(t_{1}, d\right), Q^{*}, D Q^{*}$ represents the optimal values of $t_{1}, d, F\left(t_{1}, d\right), Q, D Q$ respectively.

The behavior of the inventory level during cycle $\mathrm{T}$ is depicted in figure1. 


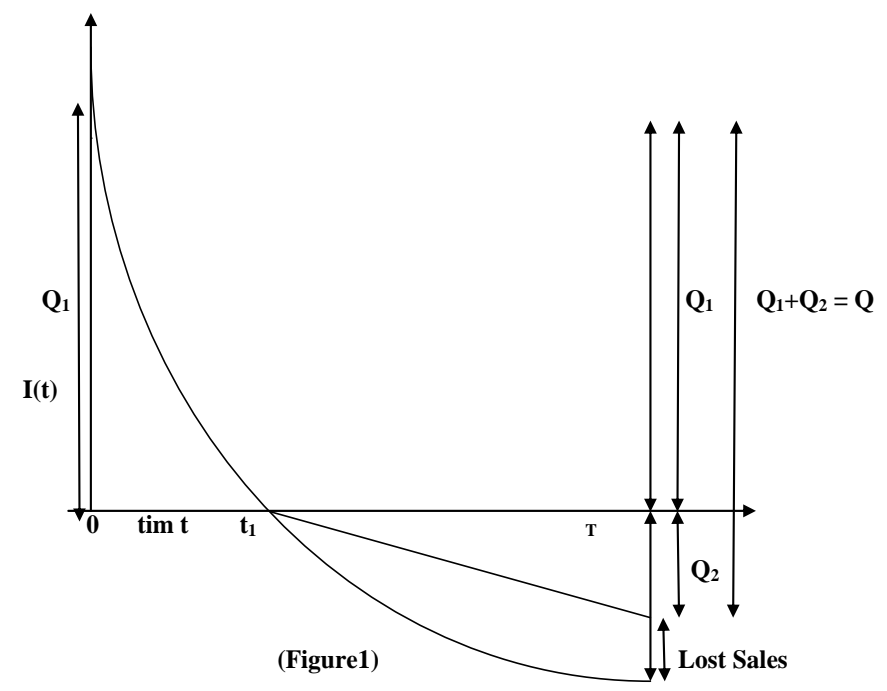

The differential equations showing the fluctuation of inventory with time $t$ are shown as below:

$$
\begin{array}{ll}
\frac{\mathrm{dI}(\mathrm{t})}{\mathrm{dt}}=-\theta \mathrm{tI}_{1}(\mathrm{t})-\mathrm{at}(\mathrm{T}-\mathrm{t}) & 0 \leq \mathrm{t} \leq \mathrm{t}_{1} \\
\frac{\mathrm{dI}(\mathrm{t})}{\mathrm{dt}}=-\frac{\mathrm{at} \alpha(\mathrm{T}-\mathrm{t})}{1+\delta(\mathrm{T}-\mathrm{t})} & \mathrm{t}_{1} \leq \mathrm{t} \leq \mathrm{T}
\end{array}
$$

With boundary condition $I\left(t_{1}\right)=0$ the solutions of these equations are given by:

$$
\begin{aligned}
& \mathrm{I}(\mathrm{t})=\mathrm{a}\left\{\left(\frac{\mathrm{Tt}_{1}^{2}}{2}-\frac{\mathrm{t}_{1}^{3}}{3}+\frac{\theta \mathrm{Tt}_{1}^{4}}{8}-\frac{\theta \mathrm{Tt}_{1}^{5}}{10}\right)+\left(-\frac{\mathrm{T}}{2}-\frac{\theta \mathrm{Tt}_{1}^{4}}{4}+\frac{\theta \mathrm{t}_{1}^{6}}{6}\right) \mathrm{t}^{2}+\frac{\theta \mathrm{T}}{8} \mathrm{t}^{4}-\frac{\theta}{15} \mathrm{t}^{5}\right\} 0 \leq \mathrm{t} \leq \mathrm{t}_{1} \\
& \mathrm{I}(\mathrm{t})=\mathrm{a}(1-\mathrm{d})^{-\mathrm{n}}\left(\frac{\left(\mathrm{t}_{1}{ }^{2}-\mathrm{t}^{2}\right)}{2 \delta}+\frac{\left(\mathrm{t}_{1}-\mathrm{t}\right)}{\delta^{2}}+\left(\frac{1+\delta \mathrm{T}}{\delta^{3}}\right)\left\{\log \left[1+\delta\left(\mathrm{T}-\mathrm{t}_{1}\right)\right]-\log [1+\delta(\mathrm{T}-\mathrm{t})]\right\}\right) \\
& \mathrm{t}_{1} \leq \mathrm{t} \leq \mathrm{T}
\end{aligned}
$$

Now if $\left.F\left(t_{1}, d\right)\right)$ is the unit time profit function then:

$\mathrm{F}\left(\mathrm{t}_{1}, \mathrm{~d}\right)=\frac{1}{\mathrm{~T}}[$ Sales revenue - purchasing cost - deterioration cost - shortage cost

-Lost sale cost - holding cost - ordering cost]

Sales revenue $\mathbf{R}=\mathrm{pQ}_{1}+\mathrm{p}(1-\mathrm{d}) \mathrm{Q}_{2}$ where

$$
\begin{aligned}
& \mathrm{Q}_{1}=\mathrm{p} \int_{0}^{\mathrm{t}_{1}} \mathrm{at}(\mathrm{T}-\mathrm{t}) \mathrm{dt} \\
& \mathrm{Q}_{1}=\frac{\mathrm{apTt} \mathrm{t}_{1}^{2}}{2}-\frac{\mathrm{apt}_{1}^{3}}{3} \\
& \mathrm{Q}_{2}=-\mathrm{I}(\mathrm{T})
\end{aligned}
$$




$$
\begin{array}{r}
\mathrm{Q}_{2}=\mathrm{a}(1-\mathrm{d})^{-\mathrm{n}}(1-\mathrm{d})\left(\frac{\left(\mathrm{T}^{2}-\mathrm{t}_{1}{ }^{2}\right)}{2 \delta}+\frac{\left(\mathrm{T}-\mathrm{t}_{1}\right)}{\delta^{2}}-\left(\frac{1+\delta \mathrm{T}}{\delta^{3}}\right) \log \left[1+\delta\left(\mathrm{T}-\mathrm{t}_{1}\right)\right]\right) \\
\mathrm{R}=\operatorname{ap}\left\{\frac{\mathrm{t}_{1}{ }^{2} \mathrm{~T}}{2}-\frac{\mathrm{t}_{1}{ }^{3}}{3}+(1-\mathrm{d})^{-\mathrm{n}}(1-\mathrm{d})\left(\frac{\left(\mathrm{T}^{2}-\mathrm{t}_{1}{ }^{2}\right)}{2 \delta}+\frac{\left(\mathrm{T}-\mathrm{t}_{1}\right)}{\delta^{2}}-\left(\frac{1+\delta \mathrm{T}}{\delta^{3}}\right) \log \left[1+\delta\left(\mathrm{T}-\mathrm{t}_{1}\right)\right]\right)\right\}
\end{array}
$$

Purchasing cost $P C=\left(Q_{1}+Q_{2}\right) C=C\left(I_{1}(0)+\left(-I_{2}(T)\right)\right.$

$$
=\mathrm{aC}\left(\frac{\mathrm{t}_{1}{ }^{2} \mathrm{~T}}{2}-\frac{\mathrm{t}_{1}{ }^{3}}{3}+\frac{\theta \mathrm{Tt}_{1}^{4}}{8}-\frac{\theta \mathrm{t}_{1}^{5}}{10}+(1-\mathrm{d})^{-\mathrm{n}}\left(\frac{\left(\mathrm{T}^{2}-\mathrm{t}_{1}{ }^{2}\right)}{2 \delta}+\frac{\left(\mathrm{T}-\mathrm{t}_{1}\right)}{\delta^{2}}-\left(\frac{1+\delta \mathrm{T}}{\delta^{3}}\right) \log \left[1+\delta\left(\mathrm{T}-\mathrm{t}_{1}\right)\right]\right)\right)
$$

Deterioration Cost $\mathbf{D C}=\operatorname{ac}\left\{\frac{\theta \mathrm{Tt}_{1}{ }^{4}}{8}-\frac{\theta \mathrm{t}_{1}^{5}}{10}\right\}$

Holding Cost $\mathrm{HC}=\mathrm{h} \int_{0}^{\mathrm{t}_{1}} \mathrm{I}(\mathrm{t}) \mathrm{dt}=\mathrm{ah}\left(\frac{\mathrm{Tt}_{1}{ }^{3}}{3}-\frac{\mathrm{t}_{1}{ }^{4}}{4}-\frac{\theta \mathrm{Tt}_{1}{ }^{5}}{15}-\frac{\theta \mathrm{t}_{1}{ }^{6}}{18}\right)$

Shortage cost $\mathbf{S C}=$ as $\left(\frac{\mathrm{T}^{3}}{6}-\frac{\mathrm{t}_{1}{ }^{2} \mathrm{~T}}{2}+\frac{\mathrm{t}_{1}{ }^{3}}{3}\right)$

Lost Sale Cost LSC $=\operatorname{al} \int_{\mathrm{t}_{1}}^{\mathrm{T}}\left(\mathrm{t}(\mathrm{T}-\mathrm{t})-\frac{\alpha \mathrm{t}(\mathrm{T}-\mathrm{t})}{1+\delta(\mathrm{T}-\mathrm{t})}\right) \mathrm{dt}$

$$
=\operatorname{al}(1-\mathrm{d})^{-\mathrm{n}}\left\{\left(\frac{\mathrm{t}_{1}}{\delta^{2}}+\frac{\mathrm{t}_{1}^{2}}{2 \delta}+\left(\frac{1+\delta \mathrm{T}}{\delta^{3}}\right) \log \left[1+\delta\left(\mathrm{T}-\mathrm{t}_{1}\right)\right]-\frac{\mathrm{T}^{2}}{2 \delta}-\frac{\mathrm{T}}{\delta^{2}}\right\}+\mathrm{l}\left(\frac{\mathrm{T}^{3}}{6}-\frac{\mathrm{t}_{1}{ }^{2} \mathrm{~T}}{2}+\frac{\mathrm{t}_{1}{ }^{3}}{3}\right)\right.
$$

\section{Ordering Cost $\mathrm{OC}=\mathrm{A}$}

Total Profit per Unit Time

$$
\begin{aligned}
& \mathrm{F}\left(\mathrm{t}_{1}, \mathrm{~d}\right)=\frac{\mathrm{a}}{\mathrm{T}}\left\{\mathrm{p}\left\{\frac{\mathrm{t}_{1}{ }^{2} \mathrm{~T}}{2}-\frac{\mathrm{t}_{1}{ }^{3}}{3}+(1-\mathrm{d})^{-\mathrm{n}}(1-\mathrm{d})\left(\frac{\left(\mathrm{T}^{2}-\mathrm{t}_{1}{ }^{2}\right)}{2 \delta}+\frac{\left(\mathrm{T}-\mathrm{t}_{1}\right)}{\delta^{2}}-\left(\frac{1+\delta \mathrm{T}}{\delta^{3}}\right) \log \left[1+\delta\left(\mathrm{T}-\mathrm{t}_{1}\right)\right]\right)\right\}\right. \\
& -\mathrm{C}\left(\frac{\mathrm{t}_{1}{ }^{2} \mathrm{~T}}{2}-\frac{\mathrm{t}_{1}{ }^{3}}{3}+\frac{\theta \mathrm{Tt}_{1}{ }^{4}}{8}-\frac{\theta \mathrm{t}_{1}{ }^{5}}{10}+(1-\mathrm{d})^{-\mathrm{n}}\left(\frac{\left(\mathrm{T}^{2}-\mathrm{t}_{1}{ }^{2}\right)}{2 \delta}+\frac{\left(\mathrm{T}-\mathrm{t}_{1}\right)}{\delta^{2}}-\left(\frac{1+\delta \mathrm{T}}{\delta^{3}}\right) \log \left[1+\delta\left(\mathrm{T}-\mathrm{t}_{1}\right)\right]\right)\right) \\
& -\mathrm{c}\left\{\frac{\theta \mathrm{Tt}_{1}^{4}}{8}-\frac{\theta \mathrm{t}_{1}^{5}}{10}\right\}-\mathrm{h}\left(\frac{\mathrm{Tt}_{1}{ }^{3}}{3}-\frac{\mathrm{t}_{1}{ }^{4}}{4}-\frac{\theta \mathrm{Tt}_{1}^{5}}{15}-\frac{\theta \mathrm{t}_{1}^{6}}{18}\right)-\mathrm{s}\left(\frac{\mathrm{T}^{3}}{6}-\frac{\mathrm{t}_{1}{ }^{2} \mathrm{~T}}{2}+\frac{\mathrm{t}_{1}{ }^{3}}{3}\right) \\
& -\mathrm{l}(1-\mathrm{d})^{-\mathrm{n}}\left\{\left(\frac{\mathrm{t}_{1}}{\delta^{2}}+\frac{\mathrm{t}_{1}{ }^{2}}{2 \delta}+\left(\frac{1+\delta \mathrm{T}}{\delta^{3}}\right) \log \left[1+\delta\left(\mathrm{T}-\mathrm{t}_{1}\right)\right]-\frac{\mathrm{T}^{2}}{2 \delta}-\frac{\mathrm{T}}{\delta^{2}}\right\}+1\left(\frac{\mathrm{T}^{3}}{6}-\frac{\mathrm{t}_{1}{ }^{2} \mathrm{~T}}{2}+\frac{\mathrm{t}_{1}{ }^{3}}{3}\right)-\mathrm{A}\right\}
\end{aligned}
$$


Order Quantity

$\mathbf{Q}=\mathrm{a}\left(\frac{\mathrm{t}_{1}{ }^{2} \mathrm{~T}}{2}-\frac{\mathrm{t}_{1}{ }^{3}}{3}+\frac{\theta \mathrm{Tt}_{1}{ }^{4}}{8}-\frac{\theta \mathrm{t}_{1}{ }^{5}}{10}+(1-\mathrm{d})^{-\mathrm{n}}\left(\frac{\left(\mathrm{T}^{2}-\mathrm{t}_{1}{ }^{2}\right)}{2 \delta}+\frac{\left(\mathrm{T}-\mathrm{t}_{1}\right)}{\delta^{2}}-\left(\frac{1+\delta \mathrm{T}}{\delta^{3}}\right) \log \left[1+\delta\left(\mathrm{T}-\mathrm{t}_{1}\right)\right]\right)\right)$

\section{SOLUTION PROCEDURE}

In the model unit time profit is a function of two variables $t_{1}$ and $d$. To find out the optimal solution

$\frac{\partial \mathrm{F}\left(\mathrm{t}_{1}, \mathrm{~d}\right)}{\partial \mathrm{t}_{1}}=0 \frac{\partial \mathrm{F}\left(\mathrm{t}_{1}, \mathrm{~d}\right)}{\partial \mathrm{d}}=0$

The optimal values of $t_{1}$ and $d$ are obtained by solving these equations simultaneously provided $\frac{\partial^{2} \mathrm{~F}\left(\mathrm{t}_{1}, \mathrm{~d}\right)}{\partial \mathrm{t}_{1}^{2}} \cdot \frac{\partial^{2} \mathrm{~F}\left(\mathrm{t}_{1}, \mathrm{~d}\right)}{\partial \mathrm{d}^{2}}-\left(\frac{\partial^{2} \mathrm{~F}\left(\mathrm{t}_{1}, \mathrm{~d}\right)}{\partial \mathrm{t}_{1} \partial \mathrm{d}}\right)^{2}>0$

\section{NUMERICAL ILLUSTRATION}

$\mathrm{T}=6$ months, $\theta=0.009, \delta=2$ units, $\mathrm{p}=100, \mathrm{~s}=0.9 \mathrm{rs} /$ unit, $\mathrm{l}=1.2 \mathrm{rs} /$ unit, $\mathrm{a}=600, \mathrm{~A}=200 \mathrm{rs} /$ order $\mathrm{h}=3.2 \mathrm{rs} /$ unit, $\mathrm{n}=3, \mathrm{C}=26, \mathrm{c}=5$.

Applying the solution procedure described above the optimal values obtained is as follows: $\mathrm{t}_{1} *=4.6750, \mathrm{~d}^{*}=0.628, \mathrm{~F}^{*}\left(\mathrm{t}_{1}, \mathrm{~d}\right)=227996 \mathrm{rs}, \mathrm{Q}^{*}=40186.6$ units, $\mathrm{DQ} \mathrm{Q}^{*}=728.68$ units

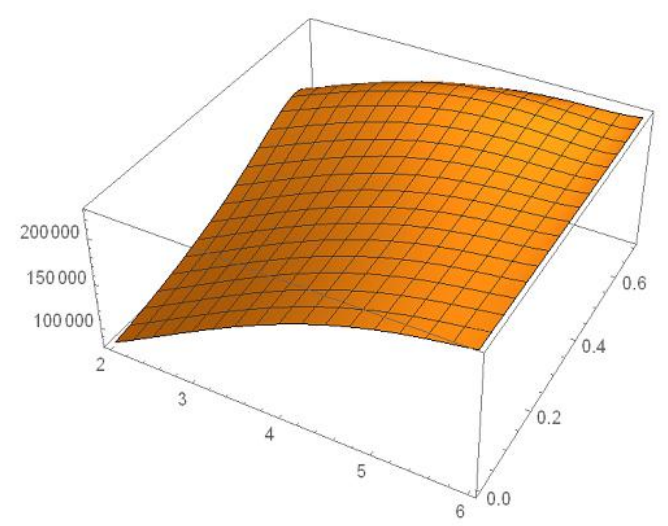

3D Graph shows the concavity of the unit time profit function $F\left(t_{1}, d\right)$

Effects of parameters, a \& p) on Total Profit per Unit Time

From the numerical Illustration given above, the effects of changing different parameters like h, a $\&$ p on time $\mathrm{t}_{1}{ }^{*}$ and discount on unit selling price $\mathrm{d}^{*}$ total profit per unit time $\mathrm{F}^{*}\left(\mathrm{t}_{1}, \mathrm{~d}\right)$ are studied.

Effects of parameter" $\delta$ "on Total Profit per Unit Time

\begin{tabular}{|l|l|l|l|l|}
\hline$\%$ variation in $\delta$ & $\delta$ & $\mathrm{t}_{1}$ & $\mathrm{~d}$ & $\mathrm{~F}\left(\mathrm{t}_{1}, \mathrm{~d}\right)$ \\
\hline$-20 \%$ & 1.6 & 4.2857 & 0.628 & 234912 \\
\hline$-15 \%$ & 1.7 & 4.395599 & 0.628 & 232716 \\
\hline$-10 \%$ & 1.8 & 4.496241 & 0.628 & 230872 \\
\hline$-5 \%$ & 1.9 & 4.589017 & 0.628 & 229315 \\
\hline $0 \%$ & 2 & 4.6750 & 0.628 & 227996 \\
\hline $5 \%$ & 2.1 & 4.75514 & 0.628 & 226874 \\
\hline $10 \%$ & 2.2 & 4.83019 & 0.628 & 225917 \\
\hline $15 \%$ & 2.3 & 4.900824 & 0.628 & 225099 \\
\hline $20 \%$ & 2.4 & 4.9676436 & 0.628 & 224400 \\
\hline
\end{tabular}

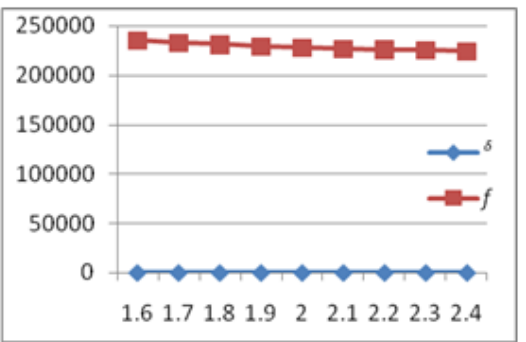

(Tablel)Variation in $\mathrm{F}\left(\mathrm{t}_{1}, \mathrm{~d}\right)$ with the

variation in $\delta$

Effects of parameter "h" on Total Profit per Unit Time 
Optimal Inventory Policy for Deteriorating Items with Seasonal Demand under the Effect of Price

\begin{tabular}{|c|c|c|c|c|}
\hline$\%$ variation inh & $\mathrm{h}$ & $\mathrm{t}_{1}$ & $\mathrm{~d}$ & $\mathrm{~F}\left(\mathrm{t}_{1}, \mathrm{~d}\right)$ \\
\hline$-20 \%$ & 2.56 & 4.72034 & 0.628 & 232603 \\
\hline$-15 \%$ & 2.72 & 4.70965 & 0.628 & 231447 \\
\hline$-10 \%$ & 2.88 & 4.698534 & 0.628 & 230294 \\
\hline$-5 \%$ & 3.04 & 4.686999 & 0.628 & 229144 \\
\hline $0 \%$ & 3.2 & 4.6750 & 0.628 & 227996 \\
\hline $5 \%$ & 3.36 & 4.66256 & 0.628 & 226850 \\
\hline $10 \%$ & 3.52 & 4.64964 & 0.628 & 225708 \\
\hline $15 \%$ & 3.68 & 4.6362 & 0.628 & 224568 \\
\hline $20 \%$ & 3.84 & 4.62232 & 0.628 & 223432 \\
\hline
\end{tabular}

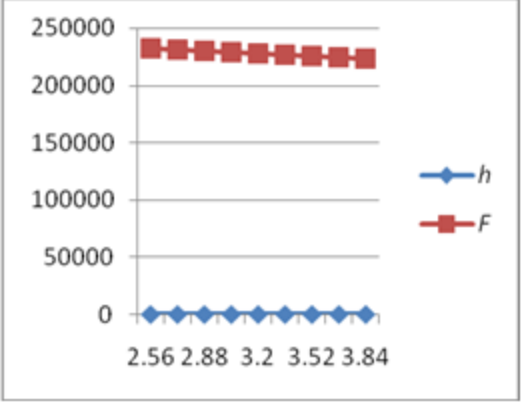

(Table 2) Variation in $F\left(t_{1}, d\right)$ with the variation in $h$

Effects of "a" on Total Profit per Unit Time

\begin{tabular}{|c|c|c|}
\hline \% variation in a & \multicolumn{1}{|l|}{ a } & $\mathrm{F}\left(\mathrm{t}_{1}, \mathrm{~d}\right)$ \\
\hline$-20 \%$ & 480 & 182397 \\
\hline$-15 \%$ & 510 & 193796 \\
\hline$-10 \%$ & 540 & 205196 \\
\hline$-5 \%$ & 570 & 216596 \\
\hline $0 \%$ & 600 & 227996 \\
\hline $5 \%$ & 630 & 239395 \\
\hline $10 \%$ & 660 & 250795 \\
\hline $15 \%$ & 690 & 262195 \\
\hline $20 \%$ & 720 & 273595 \\
\hline
\end{tabular}

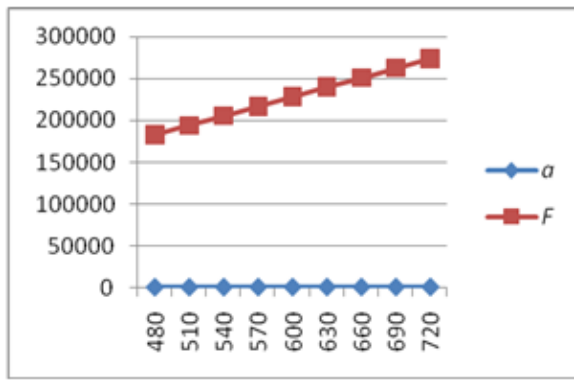

(Table 3) Variation in $F\left(t_{1}, d\right)$ with the variation in a

Effects of parameter "p" on Total Profit per Unit Time

\begin{tabular}{|c|c|c|c|c|}
\hline $\begin{array}{c}\% \\
\text { change } \\
\mathrm{p}\end{array}$ & $\mathrm{p}$ & $\mathrm{t}_{1}$ & $\mathrm{~d}$ & $\mathrm{~F}\left(\mathrm{t}_{1}, \mathrm{~d}\right)$ \\
\hline-20 & 80 & 5.40314 & 0.529944 & 147410 \\
\hline-15 & 85 & 5.41125 & 0.56317 & 166329 \\
\hline-10 & 90 & 5.05992 & 0.586667 & 185864 \\
\hline-5 & 95 & 4.8519 & 0.608421 & 206421 \\
\hline 0 & 100 & 4.675 & 0.628 & 227996 \\
\hline 5 & 105 & 4.50939 & 0.645714 & 250725 \\
\hline 10 & 110 & 5.88216 & 0.661818 & 258042 \\
\hline 15 & 115 & 5.90164 & 0.676522 & 276162 \\
\hline 20 & 120 & 5.91672 & 0.69 & 294259 \\
\hline
\end{tabular}

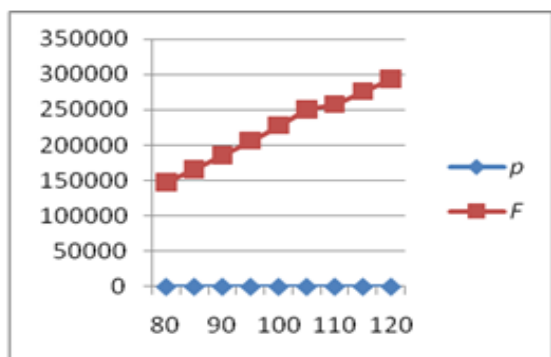

(Table 4) Variation in $F\left(t_{1}, d\right)$ with the variation in $p$

Sensitivity Analysis

\begin{tabular}{|c|c|c|c|c|}
\hline parameter & $\%$ change & $\%$ change $t_{1}$ & \%change d & \%change $\mathrm{F}\left(\mathrm{t}_{1}, \mathrm{~d}\right)$ \\
\hline \multirow[t]{4}{*}{$\mathbf{a}$} & -20 & 0 & 0 & -0.1999 \\
\hline & -10 & $\mathbf{0}$ & $\mathbf{0}$ & -0.1 \\
\hline & 10 & $\mathbf{0}$ & $\mathbf{0}$ & 0.0999 \\
\hline & 20 & $\mathbf{0}$ & $\mathbf{0}$ & 0.1999 \\
\hline \multirow[t]{4}{*}{$\mathbf{h}$} & -20 & 0.00969 & $\mathbf{0}$ & 0.0202 \\
\hline & -10 & 0.00503 & $\mathbf{0}$ & 0.1007 \\
\hline & 10 & -0.0054 & $\mathbf{0}$ & -0.010035 \\
\hline & 20 & -0.01128 & $\mathbf{0}$ & -0.02001 \\
\hline \multirow[t]{4}{*}{8} & -20 & -0.08327 & $\mathbf{0}$ & 0.0303 \\
\hline & -10 & -0.03824 & $\mathbf{0}$ & 0.01261 \\
\hline & 10 & $\mathbf{0 . 0 3 3 1 7}$ & $\mathbf{0}$ & -0.009118 \\
\hline & 20 & 0.0625 & $\mathbf{0}$ & -0.01577 \\
\hline \multirow[t]{4}{*}{$\mathbf{p}$} & -20 & 0.1557 & -0.15614 & -0.3534 \\
\hline & -10 & 0.08233 & -0.06581 & -0.18479 \\
\hline & 10 & 0.25821 & $\mathbf{0 . 0 5 3 8 5}$ & 0.13178 \\
\hline & 20 & 0.2656 & 0.09872 & 0.290632 \\
\hline
\end{tabular}

(Table 5) 


\section{OBSERVATIONS}

1. From table (1) it is observed that as the rate of backlogging $(\delta)$ decreases, the unit time profit of The system increases.

2. From table (2) it is observed that as holding cost decreases the unit time profit of the system Increases

3. From table (3) it has been noticed that an increment in demand coefficient (a) left unchanged d $\& \mathrm{t}_{1}$ and increases the unit time profit of the system.

4. Table (4) reveals that as the selling price (p) increases, the unit time profit of the system also 5. From sensitivity table (5) it has been seent ${ }_{1}$ is not sensitive to (a) but it shows small sensitivity to $(\delta),(\mathrm{h}) \&(\mathrm{p})$.d is not sensitive to $(\mathrm{a}),(\delta) \&(\mathrm{~h})$ but it is sensitive to selling price $(\mathrm{p}) . \mathrm{F}\left(\mathrm{t}_{1}, \mathrm{~d}\right)$ is Small sensitive (a), $(\delta) \&(h)$ and shows more sensitivity to selling price $(\mathrm{p})$.

\section{SPECIAL CASE OF THE MODAL (NO DISCOUNT $\mathrm{d}=0$ )}

In this case for no discount $(\mathrm{d}=0)$, the profit function $\mathrm{F}\left(\mathrm{t}_{1}, \mathrm{~d}\right)$ becomes a function of single variable $\mathrm{t}_{1}$ that is $\mathrm{F}\left(\mathrm{t}_{1}\right)$. The solution procedure to find out the optimal values is given by $\frac{d F\left(\mathrm{t}_{1}\right)}{\mathrm{dt}_{1}}=0$ provided $\frac{\mathrm{d}^{2} \mathrm{~F}\left(\mathrm{t}_{1}\right)}{\mathrm{dt}_{1}^{2}}<0$ .This gives optimal values as follows: $\mathrm{t}_{1}^{*}=5.7864, \mathrm{~F}^{*}\left(\mathrm{t}_{1}\right)=219980, \mathrm{Q}^{*}=22620.1 \& \mathrm{DQ}^{*}=1037.38$

\section{IV.Conclusion}

In the present paper an inventory model is developed with realistic features of seasonal quadratic demand which starts with zero and end with zero and end with zero, time dependent deterioration, partial backlogging. The most important feature of the modal is the declaration of price discount at the start of shortage period so that demand is boosted in this period and more customers will be willing to wait for the next replenishment. Thus in turn the discount on unit selling price increases optimum total profit per unit time. A numerical illustration is given to describe the model. Special case for no discount case for the model is discussed and the numerical illustration with no discount shows that given optimal price discount at the start of shortage period is profitable. Effect of some parameters involved in the problem on optimal discount and total profit per unit has been discussed through tables and graphs. Sensitivity analysis with respect to above parameters has been carried out. Results noticed in tables and graphs are suitable to real situations. The model could be useful in retail business of seasonal products where partial backlogging occurs. Declaration of discount on unit selling price is the key factor to enhance the demand and to increases the total back ordered quantity. The present study can be further extended for some other factor useful for inventory problems.

\section{References}

[1] Ardalan, A. (1994): Optimal prices \& Order quantities when temporary price discounts result on increase in demand, European Journal of Operational Research, 72(1), 52-61.

[2] Bhunia et al (2009): An application of tournament genetic algorithm in a marketing, oriented economic production lot size model for deteriorating items, Int.J. Production Economics, 119,112-121.

[3] Cardenas-Barron, L.E, Smith, N.R. and Goyal, S.K. (2010): Optimal Order size to take advantage of a one-time discount offer with allowed backorders, Applied Mathematical Modeling, 34, 1642-1652.

[4] Chakarbarty et al (1998): An EOQ modal for items with weibull distribution deterioration shortage and trended demand, Computers and Operation Research,25,649-657

[5] Chang, H. J. and Dye, C. Y. (1999): An EOQ model for deteriorating items with time varying demand and partial backlogging, Journal of the Operational Research Society, 50, 1176-1182.

[6] Covert, R. P. and Philip, G. C. (1973): An EOQ model for items with Weibull distribution deterioration, AIIE Transactions, 5(4), 323-326.

[7] Dave, U. and Patel, L. K. (1981): (T, $\left.\mathrm{S}_{\mathrm{i}}\right)$ policy inventory model for deteriorating items with time proportional demand. Journal of Operational Research Society, 32, 137-142.

[8] Donaldson, W. A. (1977): Inventory replenishment policy for a linear trend in demand: An analytical solution, Operational Research Quarterly, 28(3), 663-670.

[9] Dye, C. Y. (2007): Joint pricing and ordering policy for a deteriorating inventory with partial backlogging, Omega, 35(2), 184-189.

[10] Garg, G., Vaish, B., and Gupta, S. (2012): An economic production lot size model with price discounting and non-instantaneous deteriorating items with ramp type production and demand rates, Int.J.Contemp.Math.Science,7(11),531-554.

[11] Geethal, K.V. and Udaykumari, R.(2016): Optimal Lot sizing policy for non-instantaneous deteriorating items with price and advertisement dependent demand under partial backlogging,int.j.appl.comput.math,107,125-138.

[12] Ghare, P. M. and Schrader, G. H. (1963): A model for exponentially decaying inventory system, International Journal of Production Research, 21, 46-49.

[13] Hsieh,T.P. and Dye,C.Y.(2017):A note on inventory policies for products with residual-life-dependent demand,43,647-658.

[14] Hsu, W. K. and Yu, H. F. (2009): An EOQ model for imperfective items under a onetime only discount, Omega, 37(5), 1018-1026.

[15] Karmakar and Chaudhary (2014): Inventory models with, ramp type demand with partial backlogging and time varying holding cost, Yugoslav Journal of operation research, 24(2), 249-266. 
[16] Khanra, S. and Chaudhuri, K. S. (2003): A note on an order-level inventory model for a deteriorating item with time dependent quadratic demand, Computers \& Operations Research, 30, 1901-1916.

[17] Manna, S. K. and Chaudhuri, K. S. (2006): An EOQ model with ramp type demand rate, time dependent deterioration rate, unit production cost \& shortage, European Journal of Operational Research, 171, 557-566.

[18] Mishra (2013): An inventory model of instantaneous deteriorating items with controllable deterioration rate for time dependent demand and holding cost. Journal of Industrial Engineering and Management, 6(2), 495-506.

[19] Misra, R. B (1975b): Optimum production Inventory lot-size model for a system with deteriorating inventory, International Journal of Production Research, 13, 495-505.

[20] Mukhopadhyayet al (2005): An EOQ modal with two parameter weibull distribution deterioration and price dependent demand, International Journal of Mathematics Education in science and Technology,36,25-33.

[21] Panda, S., Saha, S. and Basu, M. (2009): An EOQ model for perishable products with discounted selling price and stock dependent demand, Central European Journal of Operational Research, 17(1), 31-53.

[22] Panda, S., Senapati, S. and Basu, M. (2008): Optimal replenishment policy for perishable seasonal products in a season with ramp type time dependent demand, Computers \& Industrial Engineering, 54, 301-314.

[23] Papachristos, S. and Skouri, K. (2003): An inventory model with deteriorating item, quantity discount, pricing and time dependent partial backlogging, International Journal of Production Economics, 83, 247-256.

[24] Philip, G. C. (1974): A generalized EOQ model for items with Weibull distribution, AIIE Transactions, 6, $159-162$.

[25] Sana, S. S. and Chaudheri, K. S. (2008): A deterministic EOQ models with delays in payments and price-discount offers. European Journal of Operational Research, 184, 509-533

[26] Shah and Acharya (2008): A Time dependent deteriorating Order level inventory model for exponentially declined demand, Applied Mathematically Science,56 (2),2795-2802.

[27] Silver, E. A. and Meal, H. C. (1969): A simple modification of the EOQ for the case of a varying demand rate, Production of Inventory Management 10, 52-65.

[28] Skouri, K., Konstantaras, I., Papachristos, S. and Ganas, I. (2009): Inventory models with ramp type demand rate, partial backlogging and Weibull deterioration rate, European Journal of Operational Research, 192, 79-72.

[29] Swaminathan, K.S. and Muniappan, P. (2015): Mathematical Model for optimum production inventory deteriorating items, Applied Mathematical Sciences, Vol. 9 (18), 2015, 895 - 900.

[30] Vaish, B., and Agarwal, D.(2012): Production lot size model for non-instantaneous deterioration with price discounting and variable demand rate, International Transactions in Mathematical Science and Computer,5(2),193-206.

[31] Vaish, B., and Garg, G.(2011): An optimal price discount policy for non-instantaneous deteriorating items with stock dependent and time decreasing demand, Journal of Mathematics Research,3,119-129. 